\title{
PRESENÇA DE MICROFONISMO COCLEAR \\ NO PEATE-CLIQUE: DIAGNÓSTICO DIFERENCIAL \\ ENTRE ESPECTRO DA NEUROPATIA AUDITIVA E PERDAS \\ AUDITIVAS COCLEARES DESCENDENTES EM CRIANÇAS
}

\author{
Presence of cochlear microphonics in click-ABR: \\ differential diagnosis between auditory neuropathy spectrum \\ disorder and steeply sloping cochlear hearing loss in children
}

Gabriela Ribeiro Ivo Rodrigues ${ }^{(1)}$, Silvia Nápole Fichino ${ }^{(2)}$, Dóris Ruthy Lewis ${ }^{(3)}$

\section{RESUMO}

Tema: diagnóstico diferencial entre espectro da neuropatia auditiva e perdas auditivas cocleares descendentes em crianças com presença de microfonismo coclear no PEATE-clique. Procedimentos: este relato de caso descreve os resultados da avaliação audiológica de duas crianças atendidas no Centro "Audição na Criança" da Divisão de Educação e Reabilitação dos Distúrbios da Comunicação da Pontifícia Universidade Católica de São Paulo (CeAC/DERDIC/PUCSP) que apresentaram microfonismo coclear no registro do PEATE-clique. As crianças foram submetidas às avaliações utilizandose o PEATE-clique, o registro das emissões otoacústicas e a avaliação audiológica tonal, com a técnica da Audiometria de Reforço Visual. Resultados: as avaliações comportamental, eletroacústica e eletrofisiológica revelaram que as crianças apresentam perda auditiva sensorioneural (coclear) com configuração descendente, de modo que a presença do microfonismo coclear no registro do PEATEclique era provavelmente gerada pela preservação da cóclea nas frequências baixas. Conclusão: os casos apresentados mostram que na ausência das emissões otoacústicas e presença do microfonismo coclear, não se deve interpretar isoladamente cada exame, para que não ocorram equívocos no diagnóstico, que pode ser confundido com o Espectro da Neuropatia Auditiva. O microfonismo coclear pode aparecer em outras condições, tais como em perdas auditivas cocleares descendentes.

DESCRITORES: Potenciais Evocados; Perda Auditiva Neurossensorial; Cóclea

(1) Fonoaudióloga do Centro "Audição na Criança" da Divisão de Educação e Reabilitação dos Distúrbios da Comunicação da Pontifícia Universidade Católica de São Paulo, CeAC/DERDIC/PUC-SP, São Paulo, SP; Mestre em Fonoaudiologia pela Pontifícia Universidade Católica de São Paulo; Doutoranda do Programa de Estudos Pós-Graduados em Fonoaudiologia da Pontifícia Universidade Católica de São Paulo.

(2) Fonoaudióloga do Centro "Audição na Criança" da Divisão de Educação e Reabilitação dos Distúrbios da Comunicação da Pontifícia Universidade Católica de São Paulo, CeAC/DERDIC/PUC-SP, São Paulo, SP; Mestre em Fonoaudiologia pela Pontifícia Universidade Católica de São Paulo.

(3) Fonoaudióloga do Centro "Audição na Criança" da Divisão de Educação e Reabilitação dos Distúrbios da Comunicação da Pontifícia Universidade Católica de São Paulo, CeAC/DERDIC/PUC-SP, São Paulo, SP; Professora Doutora Titular da Pontifícia Universidade Católica de São Paulo, PUC-SP, São Paulo, SP; Professora da

\section{INTRODUÇÃO}

Os potenciais evocados auditivos de tronco encefálico com o estímulo clique (PEATE-clique) vêm sendo amplamente utilizados na avaliação da função auditiva em neonatos e crianças ${ }^{1-4}$. Na suspeita do Espectro da Neuropatia Auditiva (ENA) os PEATE-clique são realizados com polaridades de estímulo condensado e rarefeito, para pesquisa do microfonismo coclear (MC) ${ }^{5}$.

Faculdade de Fonoaudiologia e do Programa de Estudos Pós-Graduados em Fonoaudiologia da Pontifícia Universidade Católica de São Paulo, PUC-SP, São Paulo, SP; Doutora em Saúde Pública pela Universidade de São Paulo.

Conflito de interesses: inexistente 
O MC é uma atividade elétrica pré-neural, ou seja, ocorre antes das sinapses das células ciliadas com o nervo auditivo, e, portanto, aparece anteriormente à onda I no registro do PEATE-clique e não há aumento de latência quando se diminui a intensidade do estímulo (função latência-intensidade) ${ }^{5}$.

Clinicamente o diagnóstico do ENA se configura pela presença das emissões otoacústicas (EOA), ausência de respostas ou importante alteração no registro do PEATE-clique desde a onda I, e presença do $M C^{6}$. Os reflexos acústicos estão quase sempre ausentes, a audiometria para tons puros varia de limiares normais a perdas severas/ profundas e a dificuldade de discriminação vocal é uma das queixas mais importantes ${ }^{7-10}$. Portanto, é comum que na presença do MC, suspeite-se de presença do ENA.

A avaliação da função das células ciliadas externas (CCE) contribui de maneira significativa para o diagnóstico diferencial do ENA. Contudo, 30\% dos indivíduos com o ENA podem apresentar comprometimento das CCE e consequente perda das EOA de modo que a ausência das EOA não exclui o diagnóstico do ENA ${ }^{11,12}$. Nestes casos a pesquisa do $\mathrm{MC}$ é determinante para se definir a funcionalidade das células ciliadas externas ${ }^{5-12}$.

Em alguns casos de perdas auditivas cocleares descendentes também é possível observar a presença do microfonismo coclear, que não deve ser confundida com o ENA. Isso porque o MC pode ser gerado pela parte mais preservada da cóclea, principalmente nas frequências baixas ${ }^{13,14}$.

Segundo as recomendações do Newborn Hearing Screening Programme ${ }^{13}$, quando o MC está presente, com PEATE-clique ausente ou alterado, deve-se suspeitar tanto do ENA como de uma perda auditiva coclear.

Diante de uma criança que não é capaz de cooperar na avaliação comportamental com procedimentos condicionados, que apresenta EOA e PEATE-clique ausentes, e da necessidade de uma intervenção terapêutica rápida e precisa, é importante que se diferencie uma perda puramente coclear do ENA ${ }^{15}$. Torna-se, portanto, de fundamental importância a realização de um diagnóstico diferencial, uma vez que a conduta adotada para a reabilitação nos casos do ENA difere dos procedimentos usualmente adotados nos casos de perda auditiva coclear ${ }^{12}$.

Com base nestas constatações, o objetivo deste relato de caso é descrever os resultados da avaliação audiológica de duas crianças atendidas no Centro "Audição na Criança" - CeAC/DERDIC/PUCSP que apresentaram $M C$ no registro do PEATE-clique, ausência de EOA e audiometria sugestiva de perdas auditivas cocleares descendentes.

\section{APRESENTAÇÃO DO CASO}

Caso 1, criança do sexo feminino, 1 ano e 10 meses, com história de nascimento prematuro, baixo peso, necessitando ficar na UTI por mais de 7 dias e ingestão de ototóxicos; e caso 2, criança do sexo feminino, 2 anos, com história de prematuridade, baixo peso e também necessitando ficar na UTI por mais de 7 dias, sendo submetida à ventilação mecânica e ototóxicos.

As crianças foram encaminhados ao Centro "Audição na Criança" - CeAC/DERDIC/PUCSP para realizar avaliação audiológica.

Foram realizadas as avaliações utilizando-se o PEATE-clique com pesquisa de $\mathrm{MC}$, registro das EOA por estímulo transiente e produto de distorção, e avaliação audiológica tonal por via aérea e óssea realizada por meio da técnica da Audiometria de Reforço Visual (VRA).

Os PEATE-clique foram registrados com 0 paciente em sono natural e o equipamento utilizado foi o Smart-EP da Intelligent Hearing Sistems (IHS). Os eletrodos foram dispostos nas mastoides direita (A1) e esquerda (A2), e os eletrodos Cz e Terra na fronte. As repostas foram registradas para cliques de polaridade condensada e rarefeita, na taxa de repetição de $27,7 \mathrm{~Hz}$.

$O$ registro das EOA evocadas por estímulo transiente e produto de distorção foi realizado com o equipamento ILO V6, de marca Otodynamics.

A audiometria com reforço visual (VRA) foi realizada por meio de condicionamento estímulo-resposta-reforço visual. Utilizou-se audiômetro AC-33, marca Interacoustics e os exames foram realizados em sala acusticamente tratada.

Para afastar a possibilidade de comprometimento da orelha média foi realizada a timpanometria, utilizando imitanciômetro da marca Kamplex, modelo AT 235H.

Este relato de caso foi analisado e aprovado pelo Comitê de Ética em Pesquisa da Pontifícia Universidade Católica de São Paulo sob protocolo de no 039/2007 e os responsáveis pelos sujeitos envolvidos assinaram o "Termo de Consentimento Livre e Esclarecido".

Os resultados foram submetidos a uma análise descritiva.

\section{RESULTADOS}

As figuras abaixo mostram os resultados dos exames audiológicos realizados com a criança do caso 1. A Figura 1 refere-se ao registro do PEATEclique, com ausência de resposta a 90 dBnNA e presença de MC bilateralmente, os dois últimos traçados de cada orelha representam o registro do 
exame com o fone de inserção obstruído (clipado) impedindo a passagem do som e, portanto, confirmando o MC.

A Figura 2 mostra o resultado ausente das EOA evocadas por estímulo transiente e produto de distorção bilateralmente.

A Figura 3 representa o audiograma, resultado da Audiometria de Reforço Visual (VRA) realizada por via aérea e via óssea.

As próximas figuras referem-se ao resultado dos exames audiológicos da criança do caso 2.

A Figura 4 mostra o resultado do PEATE-clique com resposta em $60 \mathrm{dBnNA}$ na orelha esquerda, 50 $\mathrm{dBnNA}$ na orelha direita e presença de MC bilateral.

A Figura 5 representa o resultado ausente das EOA evocadas por estímulo transiente e produto de distorção bilateralmente.

A Figura 6 representa o audiograma, resultado da Audiometria de Reforço Visual (VRA) realizada por via aérea e via óssea.

\section{DISCUSSÃO}

Estes casos levantam importantes questões que devem ser consideradas na avaliação audiológica de crianças que apresentam o MC no registro do PEATE-clique.

O ENA é caracterizado pelo comprometimento da função auditiva periférica com a integridade das CCE, normalmente indicado pela presença das EOA. No entanto, as EOA podem estar ausentes em um grande número de sujeitos com ENA e, em tais casos, o diagnóstico depende da detecção de $\mathrm{MC}$ no registro do PEATE-clique ${ }^{5}$.

A presença do $\mathrm{MC}$ tem sido considerada um sinal distintivo do ENA. No entanto, estudo recente que registrou o MC por meio da eletrococleografia transtimpânica em sujeitos com perda auditiva coclear, audição normal e ENA concluiu que a presença do $M C$ não deve ser considerada, isoladamente, um sinal distintivo do ENA, visto que o MC pode aparecer nos diferentes graus de perda auditiva coclear, incluindo até as de grau severo/profundo ${ }^{16}$.

Os casos apresentados demonstram que a ausência das EOA, com presença de $M C$ no registro do PEATE-clique, nem sempre pode determinar que o caso estudado refere-se ao ENA. O MC pode ocorrer em uma perda auditiva coclear, de configuração descendente com declives acentuados, nas quais o $\mathrm{MC}$ seria gerado pela parte mais preservada da cóclea, principalmente nas frequências baixas ${ }^{13,14}$.

Nos casos em que as EOA estão presentes, e é possível identificar o $M C$ no registro do PEATEclique ausente, o diagnóstico do ENA é mais simples. No entanto, nos casos em que as EOA estão ausentes e tem-se o registro do MC no PEATEclique, como relatado nos casos apresentados, o diagnóstico do ENA é difícil, e outras possibilidades devem ser consideradas. A bateria de exames deve ser ampliada fornecendo mais informações sobre a audição do sujeito ${ }^{17}$. Assim, na presença do MC, com PEATE-clique ausente ou alterado, deve-se suspeitar tanto do ENA, como de uma perda auditiva coclear ${ }^{13}$.

Nos casos apresentados, a possibilidade de realização da VRA por via aérea e via óssea permitiu o diagnóstico da perda auditiva coclear. No entanto, em lactentes e em crianças que não cooperam na avaliação comportamental com procedimentos condicionados, a realização do Potencial Evocado Auditivo de Tronco Encefálico por Frequência Específica e/ou de Estado Estável nas frequências de 250, 500 e $1000 \mathrm{~Hz}$ pode ajudar no diagnóstico diferencial de ENA e perda auditiva coclear descendente ${ }^{13}$.

\section{CONCLUSÃO}

Os dois casos apresentados sugerem que na ausência das EOA e presença do MC, os resultados devem ser interpretados em conjunto com uma bateria mais completa de testes, para que se possa caracterizar de forma adequada o tipo e grau da perda de audição. Ou seja, o MC pode aparecer em outras condições além do ENA, tais como em perdas auditivas cocleares descendentes.

Frente às incertezas e dificuldades frequentemente encontradas no diagnóstico audiológico infantil, os conhecimentos trazidos por estudos de casos sobre a avaliação das respostas auditivas comportamentais e eletrofisiológicas deverão afastar diagnósticos equivocados, direcionando a intervenção fonoaudiológica. 

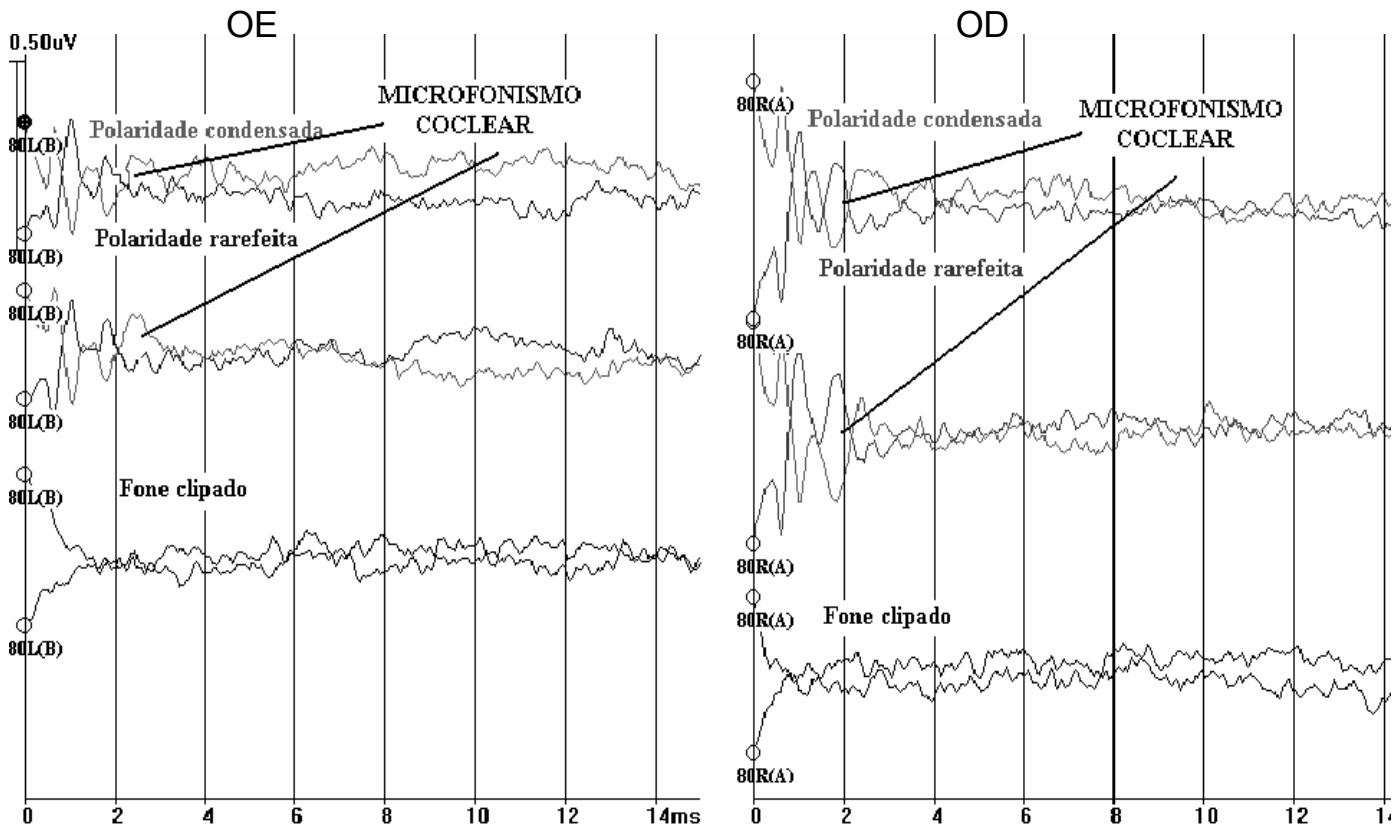

Figura 1 - Resultado do registro do PEATE-clique do caso 1

EOA transiente
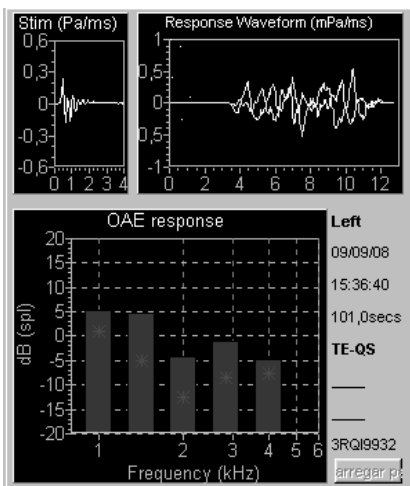

Resp $=-50,0 \mathrm{~dB}$ Noise $=14,2 \mathrm{~d}$ 日 $\mathrm{Stim}=79,6 \mathrm{~d}$ 日 NumLo $=390$ NumHi $=9$ Core
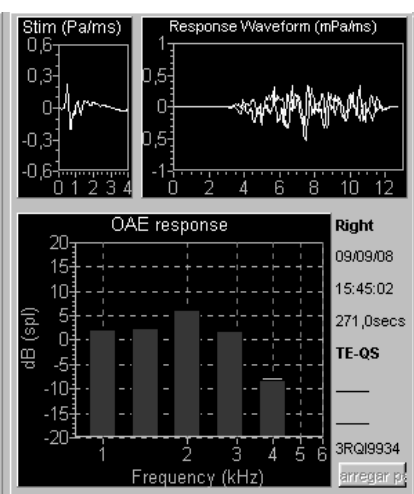

Resp $=-7,6 \mathrm{~dB} \quad$ Noise $=11,5 \mathrm{~dB} \quad \mathrm{Stim}=80,0 \mathrm{~d}$ NumLo $=1000$ NumH $=87$ Correl=-11\% Stab= 930
EOA produto de distorção
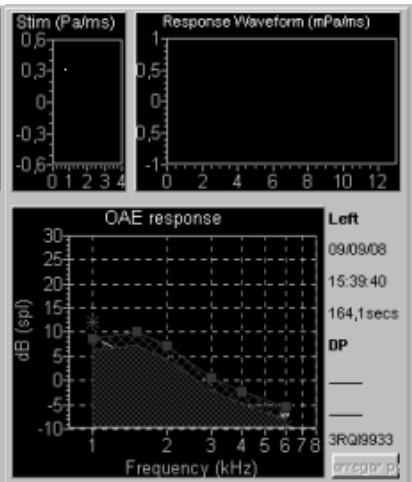

F1-65,00Bspl F2-55,00Espl OFtotol-9,0dEspl NumL $0=989$ Nunitili 755
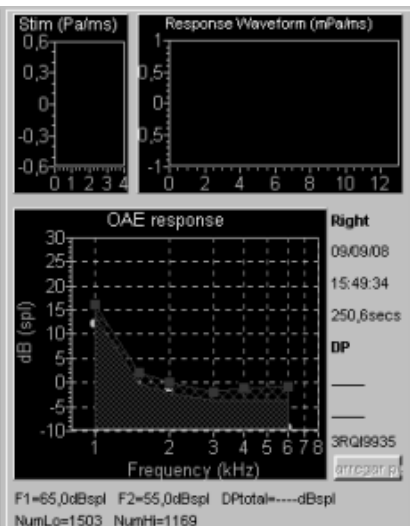

Figura 2 - Resultado das EOA transientes e produto de distorção do caso 1
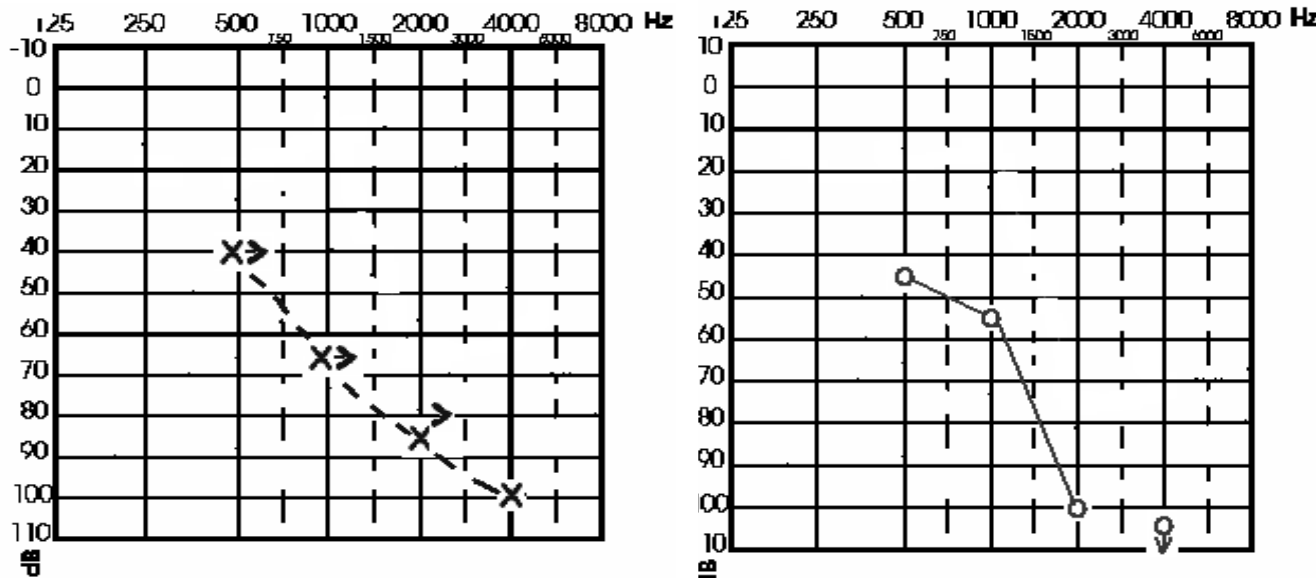

Figura 3 - Audiograma do caso 1 


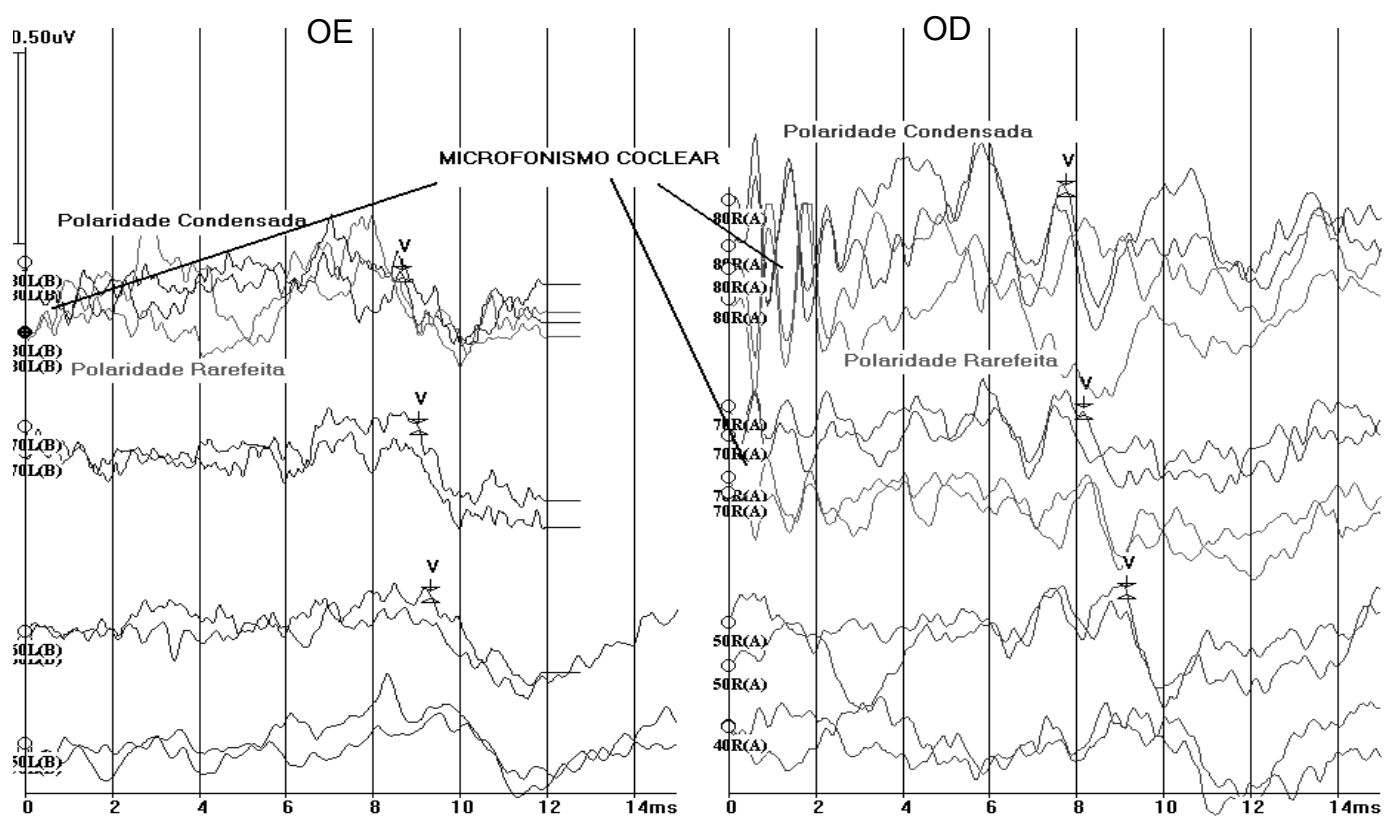

Figura 4 - Resultado do PEATE-clique do caso 2
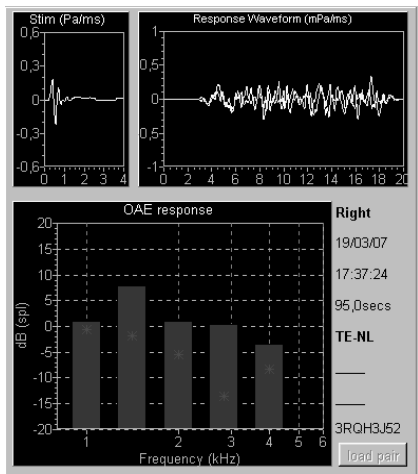

Resp $=50,0 \mathrm{~dB} \quad$ Noise $=11,3 \mathrm{~dB} \quad \mathrm{Stim}=79,6 \mathrm{~dB}$ NumLo $=414$ NumHi $=8$ Correl $=12 \% \quad$ Stab $=98 \%$
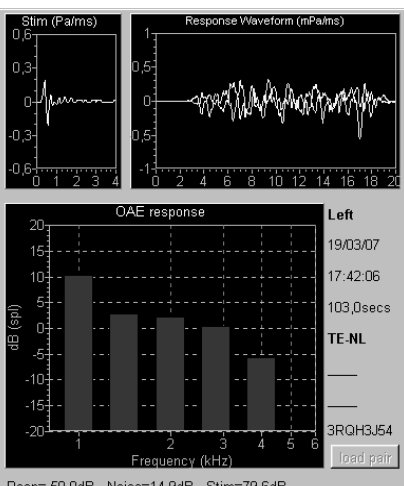

Resp $=50,0 \mathrm{~dB} \quad$ Noise $=14,9 \mathrm{~dB} \quad \mathrm{Stim}=79, \mathrm{d \textrm {dB }}$ NumLo $=484 \quad$ NumHi $=10 \quad$ Correl $=24 \% \quad$ Stab $=98 \%$
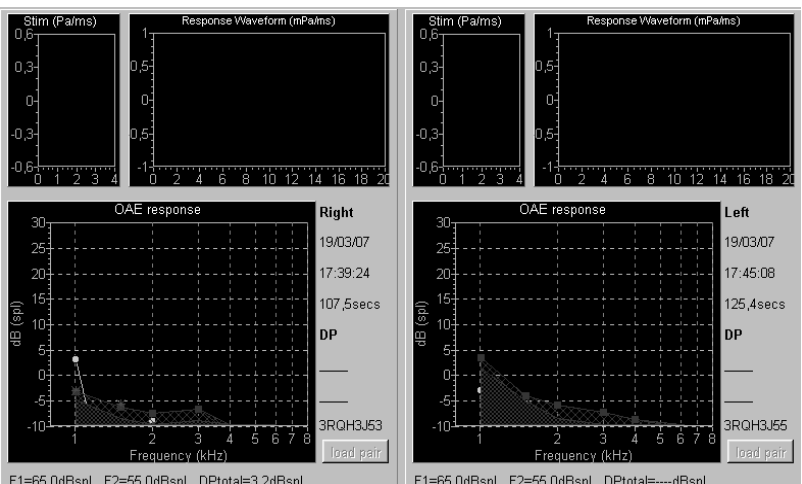

$F 1=65,0$ dBspl $F 2=55$, , OdBspl $\quad$ DPtotal= $=$ - dBspl NumLo=1243 NumHili=85

Figura 5 - Resultado das EOA transientes e produto de distorção do caso 2

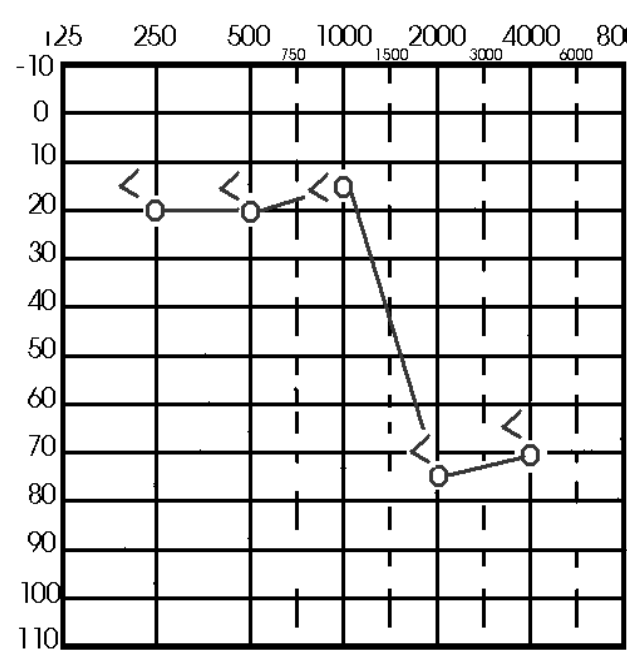

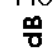

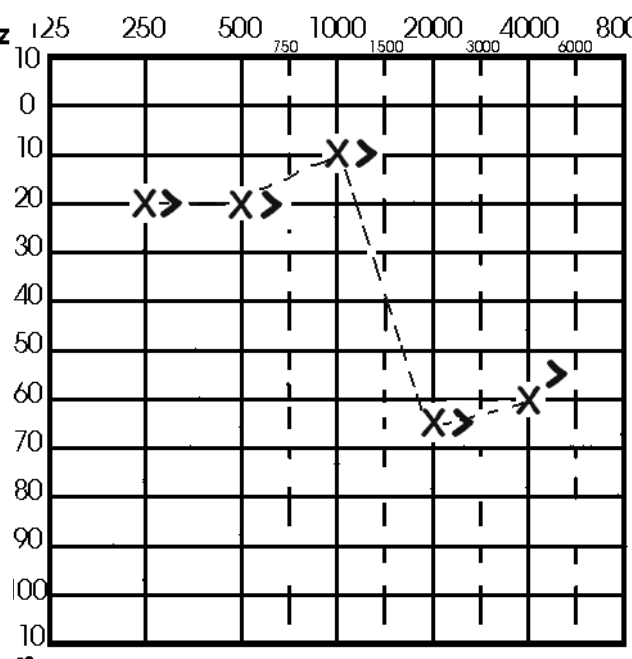

:

$8000 \mathrm{~Hz}$

Figura 6 - Audiograma do caso 2 


\begin{abstract}
Background: differential diagnosis between auditory neuropathy spectrum disorder and steeply sloping cochlear hearing loss in children with presence of cochlear microphonics for click-ABR. Procedures: this case report describes the results of the audiological evaluation for two children assisted at Centro "Audição na Criança" of Divisão de Educação e Reabilitação dos Distúrbios da Comunicação of Pontifícia Universidade Católica of São Paulo (CeAC/DERDIC/PUCSP) with presence of cochlear microphonics for click-ABR. The children are assessed by click-ABR, otoacoustic emissions and pure tone audiological evaluation, using the Visual Reinforcement Audiometry technique. Results: the test battery showed that children have sensorineural hearing loss (cochlear) with steeply sloping configuration, so that the presence of cochlear microphonics in the click-ABR was probably generated by the preservation of the cochlea in the low frequencies. Conclusion: the cases demonstrated that the absence of the otoacustic emissions, and presence of cochlear microphonics should not be interpreted as Auditory Neuropathy Spectrum Disorder, since that these conditions are similar to steeply sloping cochlear hearing loss.
\end{abstract}

KEYWORDS: Evoked Potentials; Hearing Loss, Sensorineural; Cochlea

\section{REFERÊNCIAS}

1. Luts $\mathrm{H}$, Desloovere $\mathrm{C}$, Kumar A, Vandermeersch $\mathrm{E}$, Wouters J. Objective assessment of frequencyspecific hearing thresholds in babies. Int $\mathrm{J}$ Pediatr Otorhinolaryngol. 2004; 68(7):915-26.

2. Fichino SN, Lewis DR, Fávero ML. Estudo dos limiares eletrofisiológicos das vias aérea e óssea em crianças com até 2 meses de idade. Rev Bras Otorrinolaringol. 2007; 73(2):251-6.

3. Ribeiro FM, Carvallo RM. Tone-evoked ABR in full-term and preterm neonates with normal hearing. Int J Audiol. 2008; 47(1):21-9.

4. Jiang ZD, Wilkinson AR. Normal brainstem responses in moderately preterm infants. Acta Paediatr. 2008; 97(10):1366-9.

5. Rance G, Beer DE, Cone-Wesson B, Shepherd RK, Dowell RC, King AM, et al. Clinical findings for a group of infants and young children with auditory neuropathy. Ear Hear. 1999; 20(3):238-52.

6. Deltenre P, Mansbach AL, Bozet C, Christiaens F, Barthelemy P, Paulissen D, et al. Auditory neuropathy with preserved cochlear microphonics and secondary loss of otoacoustic emissions. Audiology. 1999; 38(4):187-95.

7. Berlin Cl, Hood LJ, Morlet T, Rose K, Brashears S. Auditory neuropathy / dys-synchrony: its many forms and outcomes. Semin Hear. 2002; 23:209-14.

8. Berlin CI, Morlet T, Hood LJ. Auditory neuropathy/ dyssynchrony: its diagnosis and management. Pediatr Clin North Am. 2003; 50(2):331-40.
9. Berlin Cl, Hood LJ, Morlet T, Wilensky D, St John $\mathrm{P}$, Montgomery E, et al. Absent or elevated middle ear muscle reflexes in the presence of normal otoacoustic emissions: a universal finding in 136 cases of auditory neuropathy/dys-synchrony. J Am Acad Audiol. 2007; 18(2):187-9.

10. Shehata-Dieler W, Völter C, Hildmann A, Hildmann $\mathrm{H}$, Helms J. [Clinical and audiological findings in children with auditory neuropathy]. Laryngorhinootologie. 2007; 86(1):15-21.

11. Starr A. The neurology of auditory neuropathy. In: Sininger I, Starr A. Auditory neuropathy, a new perspective on hearing disorders. San Diego: Singular Publishing Group; 2001. p. 37-49.

12. Roush P. Auditory neuropathy spectrum disorder: evaluation and management. Hear J. 2008; 61(11):36-41.

13. Gravel J, Hood L, Lightfoot G, Mason S, Sirimanna T, Stevens J, et al. Assessment and management of auditory neuropathy / auditory dys-synchrony: recommended protocol. Newborn Hearing Screening Programme (England). 2004. Disponível em: URL: http://hearing.screening.nhs. uk/cms.php?folder=84

14. Coats AC, Martin JL. Human auditory nerve action potentials and brain stem evoked responses: effects of audiogram shape and lesion location. Arch Otol. 1977; 103(10):605-22.

15. Fávero ML, Silva FLC, Tabith Junior T, Nicastro FS, Gudmond MC, Spinelli M. Mudanças nos parâmetros do clique durante a captação do BERA. Rev Bras Otorrinolaringol. 2007; 73(1):7-11. 
16. Santarelli R, Scimemi $P$, Dal Monte E, Arslan E. Cochlear microphonic potential recorded by transtympanic electrocochleography in normallyhearing and hearing-impaired ears. Acta Otorhinolaryngol Ital. 2006; 26(2):78-95.
17. Ahmmed A, Brockbank C, Adshead J. Cochlear microphonics in sensorineural hearing loss: lesson from newborn hearing screening. Int $\mathrm{J}$ Pediatr Otorhinolaryngol. 2008; 72(8):1281-5.

DOI: 10.1590/S1516-18462009005000068

RECEBIDO EM: 27/08/2009

ACEITO EM: 25/10/2009

Endereço para correspondência:

Gabriela Ribeiro Ivo Rodrigues

Centro "Audição na Criança" - Divisão de

Educação e Reabilitação dos Distúrbios da

Comunicação PUC/SP

Rua Estado de Israel, 860

São Paulo - SP

CEP: 04022-040

E-mail: gabrielaivo@ hotmail.com 